\title{
Documenta Mathematica
}

\author{
von Alfred K. Louis, Ulf Rehmann und Peter Schneider
}

Documenta Mathematica, Journal der Deutschen Mathematiker-Vereinigung, hat seinen Betrieb aufgenommen: Die erste Ausgabe wird nun erstellt und demnächst erscheinen. Als voll elektronisches Journal hat es kurze Publikationszeit und praktisch keine Platzbeschränkungen. Es ist im Internet (World Wide Web) unter der Adresse http://www .mathematik. uni-bielefeld.de/documenta zu lesen. Weltweit kann sich jeder Leser die Artikel, die ihn interessieren, sofort nach Annahme zur Publikation nicht nur am Bildschirm ansehen, sondern auch - in gewohnter $T_{E} X$-Qualität - direkt am Arbeitsplatz oder mit dem Bereichsdrucker ausdrucken.

Die Arbeiten - aus dem gesamten Bereich der Mathematik - werden von den Autoren als $\mathrm{T}_{\mathrm{E}} \mathrm{X}$ Datei - per Electronic Mail oder auch als Diskette - bei einem der Herausgeber eingereicht, der dann die Arbeit in üblicher Weise anonym referieren läßt, um die Entscheidung über Annahme oder Ablehnung herbeizuführen. Nach Annahme einer Arbeit wird sie umgehend veröffentlicht, wenn der Autor das Recht auf Veröffentlichung an Documenta MatheMATICA übertragen hat. Die Referateorgane „Mathematical Reviews" und "Zentralblatt für Mathematik" werden die Publikationen genauso referieren, wie das bei herkömmlichen gedruckten Journalen der Fall ist. Documenta Mathematica wird als eine der führenden Zeitschriften nur mathematische Forschungsarbeiten hoher Qualität veröffentlichen.

Die Qualitätskontrolle wird durch die Herausgeber gewährleistet. Das DMV-Präsidium hat zunächst für die Dauer von vier Jahren - drei geschäftsführende Herausgeber bestellt und damit beauftragt, ein Herausgebergremium zu finden und mit den Vorbereitungen für die erste Ausgabe zu beginnen. Die geschäftsführenden Herausgeber sind A.K. Louis (Saarbrücken), U. Rehmann als technischer Herausgeber (Bielefeld) und P.Schneider (Münster). Als Herausgeber konnten gewonnen werden: D. Blasius (Los Angeles), J. Cuntz (Heidelberg), B. Fiedler (FU Berlin), F. Götze (Bielefeld), W. Hackbusch (Kiel), U. Hamenstädt (Bonn), M. Karoubi (Paris), R. Kreß (Göttingen), S. Lichtenbaum (Providence), A.S. Merkurjev (St.Petersburg), A. Nerode (Ithaca), T.Peternell (Bayreuth), W. Soergel (Freiburg), G. M. Ziegler (TU Berlin).

Die Arbeiten werden jahrgangsweise zu Bänden zusammengefaßt und als Bestandteile eines Bandes in einheitlichem Layout mit fortlaufender Seitenzahl und Inhaltsverzeichnis gestaltet. Ein solcher Jahrgang kann nach Ausdruck von den Bibliotheken gebunden und wie üblich in den Regalen aufgestellt und archiviert werden. Darüber hinaus ist auch geplant, die Jahrgänge als CD-Roms erscheinen zu lassen. Im elektronischen Angebot - also auf dem Internet und später auf den CD-Roms - werden nicht die $\mathrm{T}_{\mathrm{E}} \mathrm{X}$ -
Dateien, sondern druckfertige DVI- und PostscriptVersionen angeboten. Zusätzlich erscheint zu jeder Arbeit ein Abstract, der in HTML gestaltet ist und Stichwörter, MSC und Hyperlinks enthalten kann. Der elektronische Gesamtband, oder eine Vielzahl hiervon, kann dann nach diesen Stichwörtern schnell mit elektronischen Mitteln durchsucht werden.

Der Autor ist in der Wahl des $\mathrm{T}_{\mathrm{E}} \mathrm{X}$-Dialekts (plain $\mathrm{T}_{\mathrm{E}} \mathrm{X}, \mathrm{L}_{\mathrm{E}} \mathrm{T}, \mathcal{A}_{\mathcal{M}} \mathcal{S}-\mathrm{T}_{\mathrm{E}} \mathrm{X} \ldots$...) und der zugrundeliegenden Makro-Sammlung weitgehend frei. Das einheitliche Layout wird erreicht, indem eine kurze, von Documenta Mathematica bereitgestellte $\mathrm{T}_{\mathrm{E}} \mathrm{X}$-Datei der eigentlichen Arbeit vorangestellt wird. In diese sind Titel, Abstract sowie Name und Adresse des Autors oder der Autoren einzutragen.

Sollen Grafiken eingebunden werden, so können die üblichen $\mathrm{T}_{\mathrm{E}} \mathrm{X}$-Werkzeuge verwendet werden, oder es können Postscript-Dateien eingebunden werden. Auf diese Weise ist gewährleistet, daß problemlos auch hochqualitative Grafiken wie Zeichnungen und Photos sowohl in Schwarzweiß als auch in Farbe wiedergegeben werden können.

Eine Bemerkung zu den Verwertungsrechten an den Arbeiten: Documenta Mathematica läßt sich vom Autor nur das Recht auf Publikation übertragen. Der Autor behält das Recht an der eigenen Arbeit. Jedoch muß in einer Folgepublikation - etwa in einem Buch oder einer Werkausgabe - auf die Erstpublikation verwiesen werden, und das Publikationsrecht von Documenta Mathematica darf nicht einschränkt werden.

Platzbeschränkungen sind in Documenta MATHEMATICA nicht gegeben - neben der Schnelligkeit eine der Stärken der elektronischen Publikation. Documenta Mathematica wird dies nutzen, um Artikel mit qualitätvoller Mathematik zu publizieren, deren Attraktivität z. B. auch durch besonders sorgfältig geschriebene Einleitungen erhöht wird. Wir freuen uns auf Thre Manuskripteinsendungen!

Adresse des technischen Herausgebers:

Prof. Dr. Ulf Rehmann

Fakultät für Mathematik

Universität Bielefeld

Postfach 10 01 31, 33501 Bielefeld 